\title{
Futility: What Cool Hand Luke Can Teach the Surgical Community
}

\author{
Eric Grossman · Peter Angelos
}

Published online: 4 April 2009

(c) Société Internationale de Chirurgie 2009

When the iconic protagonist Luke Jackson, as played by Paul Newman in the 1967 classic movie Cool Hand Luke, defiantly mocked the warden in his last moments alive by uttering, "What we've got here is a failure to communicate," the fields of medicine and cinema were forever married. No other phrase can so aptly encapsulate nearly every problem associated with the medical profession, and more specifically, the inherent difficulties with the term medical futility. In this brief editorial, we attempt to review the current medical futility policies; more importantly, however, we propose an innovative approach for surgeons caring for patient's with complex problems.

In today's modern medical arena, there is nearly nothing that is truly impossible. Innumerable conditions that once proved to be uniformly fatal are now either completely reversible or at least manageable. As the fields of medicine and surgery improve, much of what was once inconceivable is now commonplace. However, simply because the technology exists to prolong life, does not imply that using such technology is always in our patients' best interests.

\section{Case report}

Mrs. A, a 63-year-old mother of three adult daughters whose husband died 3 years earlier, was transferred from a

E. Grossman $(\bowtie) \cdot$ P. Angelos

Department of Surgery, The University of Chicago Medical

Center, 5841 South Maryland Avenue, MC 5029, Chicago,

IL 60637, USA

e-mail: eric.grossman@uchospitals.edu

\section{P. Angelos}

MacLean Center for Clinical Medical Ethics, The University of Chicago, 5801 South Ellis, Chicago, IL 60637, USA community hospital in septic shock to the medical intensive care unit (MICU) of a tertiary care university medical center. Two months prior to that, she had been diagnosed with multiple myeloma, and was receiving chemotherapy and radiation. At the time of her arrival in the MICU, she was found to be hypotensive. Despite aggressive fluid resuscitation, she remained hypotensive, prompting the use of vasopressive agents. Over the next two days, her leukopenia worsened, decreasing to a nadir of 800 cells per microliter, her metabolic acidosis progressed to a lactic acid level of $6.7 \mathrm{mmol} / \mathrm{l}$ (reference: 0-2.4 mmol/l), and her blood cultures grew Gram-negative bacilli. She developed respiratory failure requiring intubation and mechanical ventilation. Her abdomen became distended, and radiographs demonstrated dilated loops of small bowel. The surgical service was consulted for a presumed diagnosis of ischemic bowel. As the attending surgeon (P.A.), I explained to her children that Mrs. A might survive if she underwent operation and that there was little chance for survival if no intervention was undertaken. The numerous risks associated with surgery, as well as the potential for death, were made clear in discussions with the family. Ultimately, I recommended an exploratory laparotomy, and Mrs. A's daughters readily consented to the operation.

Abdominal exploration revealed multiple areas of necrotic small bowel, and nearly the entire colon was necrotic. A portion of midjejunum was resected, along with the right, transverse and descending colon. The small bowel was reconstructed with a primary stapled anastomosis. An end-ileostomy was constructed, as well as a mucous fistula from the remaining sigmoid colon. After these procedures, the patient was returned to the surgical intensive care unit. When I met with the family, I informed them of the operative findings, and the likely poor prognosis. Specifically, I stated that chances for recovery were 
small; however, if Mrs. A's condition were to worsen, I thought that it would be virtually impossible for her to recover. I thought that this interchange with the family had gone well and that they appreciated the gravity of the situation. They thanked me for my frankness and honesty.

Unfortunately, during the next week Mrs. A's condition did worsen; she developed acute respiratory distress syndrome, renal failure, and eventually hepatic failure. In a subsequent family meeting, I informed the family that additional treatment would only prolong Mrs. A's suffering, and would therefore be futile; Mrs. A would likely die, no matter what was done. I recommended that aggressive treatment cease, and that comfort care measures be initiated. To my surprise, the family was quite offended by this proposal. They questioned how I, someone outside of their family, could decide when the burdens of any treatment regimen outweigh its potential benefits. To my proclamation of futility, they responded, "Futile in relation to what?" And "Futile for whom?" Sadly, after this conversation, Mrs. A's daughters became suspicious and even mistrustful of me and the surgical team, and they insisted that all treatments continue. Mrs. A remained in the intensive care unit, and members of our surgical team continued to communicate with her family regularly. The family meetings that were once constructive and positive had deteriorated to the perfunctory detailing of Mrs. A's worsening condition. One-week later, Mrs. A died in the surgical intensive care unit.

As the attending surgeon caring for Mrs. A, I have revisited her case innumerable times in my mind. Although I am saddened by her death, which I believe was the unfortunate consequence of her sepsis and ischemic bowel, I remain convinced that our surgical team provided her with the best chance of recovery. However, the disconnection between the surgical team and Mrs. A's family that was produced upon the declaration of futility, has profoundly affected me. Clinically, it was apparent that Mrs. A's condition had become unrecoverable, yet describing further treatment to her family as futile only served to isolate the family from her doctors. I have since wondered, "When is a treatment futile?"

\section{Futility in the literature}

There have been numerous published attempts at addressing this question. Earlier definitions were quantitative; in the late 1980s and early 1990s, both Blackhall [1] and Schneiderman et al. [2] defined futile options as having less than a $1 \%$ chance of success. In contrast, a decade later, Jones and McCullough defined the term much more broadly, simply asserting that the therapeutic goal of a clinical intervention is unlikely to be achieved [3]. The
Society of Critical Care Medicine (SCCM) [4] defines specific categories of futile treatment that include the probability of achieving a beneficial effect, as well as the cost of the treatment. However, because of the ambiguity of the term "futile," the SCCM cautioned against using the term. In fact, defining when a medical situation is futile has become so challenging, that in 2001, McCullough and Jones [5] published an algorithm for making such decisions. These authors felt that by honestly assessing a complex medical situation and adhering to their prescribed outline caregivers would be able to discover the "correct" answer regarding futility. It should come as no surprise that, despite the numerous definitions and rubrics regarding futility, surgeons and other medical professionals have yet to reach a consensus regarding this nebulous concept.

The myriad of difficulties associated with simply defining the term futility prompts the ensuing question: "Why has the term 'medical futility' persisted?" We believe that the concept of medical futility is the unfortunate by-product of the changing and deteriorating, doctor-patient relationship. What once resembled a pedagogical, although slightly paternalistic relationship, is now based primarily on patient autonomy, and not infrequently on health insurance limitations. To help swing the pendulum of decision-making power back toward the medical professionals, the term futility is often invoked. The consequence of declaring a situation as futile is that the patient and family members are no longer significant participants in future decision making. Schneiderman et al. [6] have reported similar sentiments, asserting that the term "medical futility" is simply a tool to increase the power of the physician over the patient, and to "repeal recent hard-gained advances in patient autonomy." Lantos et al. [7] elegantly outlined the complexities of the shifting ethical obligations of physicians, and presciently cautioned against flippantly using the term "futile" and thus abandoning one's patient. Nonetheless, declaring a situation futile, by whatever definition, does not achieve the intended goal. Patients become confused, medical professionals become frustrated, and there is further compromise of the doctorpatient relationship. In a recent review, Helft et al. warn about using the concept of "futility" as leverage in the doctor-patient relationship, and they propose that emphasis be placed on the processes for discussing futility rather than simply implementing decisions based on futility [8].

In retrospect, we believe that declaring additional treatment as "futile" marginalized Mrs. A's daughters because they felt that they were no longer actively participating in their mother's care. This, in turn, caused them to lose trust in the surgical team and ultimately created an unsettling and stressful situation for her family. 


\section{Eliminating "futility"}

It is for this reason, exactly, that we propose-rather than redefining yet again a term that seems to be immune from definition - to strike the term from our professional lexicon. The multiple variations in definitions serve only to highlight the challenges and limitations of the concept. When the medical condition is not clearly explained, or the goals of treatment are not reasonably established, the resulting discrepancies can grow and become an obstacle to patient care. Because invoking the term futility worsens communication, and lessens patient care we recommend that increased efforts be made to educate patients and their families regarding realistic expectations of the patient's disease and its prognosis. In this way, realistic patient goals can be established and readdressed as necessary. Redefining the term futility based on doctor-patient communication rather than on clinical criteria has previously been proposed by Burns and Truog [9]. Furthermore, there is growing support of the theory that any use of the term medical futility may itself be futile $[8,10]$.

As surgeons, we must educate our patients about their status, and offer the treatment that is in their best medical interest. If this recommended course of action proves to be undesirable to the patient or the family, we should take the time to understand our patient's desires and care-oriented goals. Finally, if, despite our efforts to educate, there remains disparity regarding the specifics of patient care, it is our recommendation that surgeons as a group should feel confident in their professional assessments and clinical decisions. Rather than refer to treatment options as futile, the term medically and surgically inappropriate should be invoked. This most accurately describes the clinical scenario and the surgeon's professional opinion.

Whether a better outcome could have been achieved had the discussion with Mrs. A's family been framed differently is impossible to know. However, in order to strengthen and preserve the doctor-patient relationship, improved communication and patient relations must be sought.

In conclusion, as surgeons we care for the sickest and often most desperate patients. Our patients look to us for hope and honesty. The only ethical option we have is to communicate with our patients, honestly disclose their status and prognosis, offer our best medical advice, and never hide behind the term futility.

\section{References}

1. Blackhall LJ (1987) Must we always use CPR? N Engl J Med 317:1281-1285

2. Schneiderman LJ, Jecker NS, Jonsen AR (1990) Medical futility: its meaning and ethical implications. Ann Intern Med 112:949954

3. Jones JW, McCullough LB (2002) Futility and surgical intervention. J Vasc Surg 35:1305

4. Society of Critical Care Medicine (1997) Consensus statement of the Society of Critical Care Medicine's Ethics Committee regarding futile and other possibly inadvisable treatments. Crit Care Med 25:887-891

5. McCullough LB, Jones JW (2001) Postoperative futility: a clinical algorithm for setting limits. Br J Surg 88:1153-1154

6. Schneiderman LJ, Jecker NS, Jonsen AR (1996) Medical futility: response to critiques. Ann Intern Med 125:669-674

7. Lantos JD, Singer PA, Walker RM et al (1989) The illusion of futility in clinical practice. Am J Med 87:81-84

8. Helft PR, Siegler M, Lantos J (2000) The rise and fall of the futility movement. N Engl J Med 343:293-296

9. Burns JP, Truog RD (2007) Futility: a concept in evolution. Chest 132:1987-1993

10. Moseley KL, Silveira MJ, Goold SD (2005) Futility in evolution. Clin Geriatr Med 21:211-222, x 